\title{
Causal Understanding of Limited Adoption of Cloud Computing by Customers
}

\author{
Neeta Sharma \\ Research Scholar \\ Noida International University \\ Greater Noida, UP
}

\author{
Mayank Singh, PhD \\ Associate Professor \\ Krishna Engineering College \\ Ghaziabad, UP
}

\author{
Anuranjan Misra, PhD \\ Professor \\ Noida International University, \\ Greater Noida, UP
}

\begin{abstract}
As Cloud Computing is an advanced technology with better functionality offered internet services on rent base with many advantages such as scalability, flexibility, integration, and cost reduction. In addition it provides an advance virtual storage for organizations to deploy their applications for perform the operations. With respect of all benefits of Cloud Computing technology the organizations are still reluctant to adopt Cloud Computing services due to security and threats concerns. Security is one of the main challenges that encumber the growth of Cloud Computing. On the other hand, cloud service providers (CSP) endeavor to reduce the risks over the clouds and increase their reliability between them and cloud users. In this paper, we have tried to investigate the reason for of limited adoption of Cloud Computing by the consumers and findings.
\end{abstract}

\section{Keywords}

Cloud Computing, Security \& Threats, Privacy, Availability

\section{INTRODUCTION}

The evolution and expansion of electronic services requires continuous enhancement in the term of infrastructure. In this context, Cloud Computing offers a comparatively cost effective (pay as per usage) scalable alternative to wherever to use infrastructure. Cloud Computing refers to hosted services online. These services are access via the internet which is symbolically depicted as a 'cloud' and a model which demonstrates a promising future for the consumers.

Cloud Computing offers various services on different layers such as SaaS (Software as a Service is also known as Software layer and Gmail is best example of this), PaaS (Platform as a Service is responsible for IT services such as Operating System or an application framework and customer also installed or developed additional software) and IaaS (Platform as a Service is responsible for IT services like Operating System or an application framework and customer also installed or developed additional software. With service layers Cloud Computing also provides deployment models. Deployment models are private cloud (a type of cloud where parts of computing resources can also be reserved and dedicated for one organization/customer only is known as private cloud computing), public cloud (a type of cloud where parts of computing resources can be shared to the public), community cloud (a type of cloud where parts of computing resources can be shared infrastructure for specific community and hybrid cloud (A type of cloud where parts of computing resources can also be shared for by the two and more organizations). On the conclusion we can say that Cloud Computing is delivers all types of hardware and softwares [1].
However, using services over the cloud is accompanied with much doubt mostly about security issues. IDC survey report shows that security is the utmost concern of cloud users [2]. Moving and storing data over a network using a third party (CSP) is tough decision to be approved. Nevertheless, Cloud Computing should assure for good performance, availability and secure storage and transmission. Furthermore organizations are more reluctant to adopt Cloud Computing for move their business or sensitive data where specific infrastructure, privacy level, cost level and many other concerns will be unknown. So, there is highly requiring serious action to covers their week points. This research paper present a conceptual study of the security issues such as data, network and web-based in Cloud Computing environment.

\section{LITERATURE REVIEW}

In the paper "Study of Security Issues in Cloud Computing(June 2015)", Versha et al discuss the tenets of this new and emerging technology cloud computing that provides shared resources and services at decreased cost of hardware and software along with some associated security issues while using services of cloud. Further, authors emphasis on the feature of multi tenancy while also discussing the security issues of data on the cloud discovered by CSA. Authors conclude that security issue in cloud computing can be decreased by modifying or designing a strong and robust architecture for multi tenancy [3].

In the paper "A Survey of Cloud Authentication Attacks and Solution Approaches (October 2014)", B. Sumitra et al. have analysed that, it is an evolving computing model that offers great potential but providers of this fast growing technology requires to address many issues such as virtualization, application security, identity management, access control and authentication. This paper mainly focuses on probably all types of authentication attacks that restrict legitimate users' access to services and propose possible mitigation countermeasures in the cloud. Discussing about various authentication attacks such as eavesdropping, man-inthe-middle, cookie poisoning, replay, Session hijacking, malware injection, DoS, insider attacks etc the authors suggest that user authentication mechanism should be strong enough to protect cloud from various types of attacks [4].

In the paper "A Survey on Security Issues and Solutions At different layers of Cloud Computing (Feb 2013)" Chiraj Modi el have opined that, since cloud services are delivered using classical network protocols and formats over the Internet, implicit vulnerabilities existing in these protocols as well as threats introduced by newer architectures raise many security and privacy concerns. The factors affecting Cloud Computing adoption, vulnerabilities and attacks, and identify relevant solution directives to strengthen security and privacy 
in the cloud environment [5].

In the paper "An overview and Study of Security Issues \& Challenges in Cloud Computing (September 2012)" Rajesh Piplode el discovered through survey that the major obstacle for adoption of cloud is concern for security. They list out ten security concerns in details and some solutions for security issues in Cloud Computing including Investigation Support, Network Security, Encryption Algorithm, Backup, and Customer satisfaction. Most importantly, the authors have proposed a Security Management Model called CMM which describes twenty recommended security management models and their requirements for cloud computing that cloud service providers should definitely consider as they develop or refine their compliance programs [6].

In the paper "A Brief Survey on Architecture, Challenges and Security Benefit in Cloud Computing (February 2012)" Ajith Singh .N el have expressed their worries about bigger companies that still lack the conviction that their bulk data will be maintained with the utmost privacy over the internet. This concern can only be resolved by drafting strict rules for maintaining data privacy over Cloud Computing and strict adherence to these rules. Invention of internet changed the way we use of computer and cloud is going to change that to other level [7].

In the paper "Cloud Hooks: Security and Privacy Issues in Cloud Computing (2011)", Wayne A. Jansen claims that Cloud computing can be implemented entirely within an organizational computing environment as private cloud. Cloud Computing offers a chance for reliable online digital storage of files, often quite helpful for users accessing the internet from mobile phones or internet cafés, and without large storage devices. Cloud Computing transfers much of the processing required to use web applications away from the browser as processing is done "in the cloud" in the distributed infrastructure (e.g. servers) of the Cloud Computing service. With these advantages of Cloud computing it also faces security threats vulnerabilities. In the paper, author identifies key issues, which are believed to have long-term significance such as adoption, vulnerabilities and attacks, and also identifies prevalent in cloud computing security and privacy, based on documented and exhibited weaknesses [8].

In the paper "A survey on security issues in service delivery models of Cloud Computing (January 2010)", S. Subhashini et al express their fear that the architecture of cloud poses a threat to the security of the existing technologies when deployed in a cloud environment. Cloud service users need to be vigilant in understanding the risks of data breaches in this new environment. In this paper, a survey of the different security risks that pose a threat to the cloud is presented. This paper brings forth the result of the survey on different security issues caused due to the nature of the service delivery models of a Cloud Computing system [9].

In the paper "Security Guidance for Critical Areas of Focus in Cloud Computing (April 2009)", Jeff Bardin et al observe that, Phase one of the Internet was connectivity, with Cloud Computing we are leveraging the connectivity to optimize the utility of computing. Some deride the cloud as nothing new and in many respects they are correct but Cloud Computing is having its moment in the sun, as the concept of utilizing computing as an on-demand subscription creates operating and economic efficiencies. They believe Cloud Computing to be a very important trend that in many ways is beginning to fulfill the early promise of the Internet and will create unanticipated change in business with its ubiquitous adoption. All intentions are to provide security practitioners with a comprehensive roadmap for being proactive in developing secure and positive relationships with cloud providers. To a great extent, this guidance is also quite relevant to the cloud provider to improve the quality and security of their service offerings. As with any initial venture, there will certainly be some areas that we could improve upon. We will quite likely modify the number of domains and change the focus of some areas of concern [10].

\section{BACKGROUND}

Cloud Computing is an advanced technology with better functionality offered for internet services on rent basis, with many advantages such as scalability, flexibility, integration, and cost reduction. In addition, it provides an advanced virtual storage for organizations to deploy their applications to perform the operations. Cloud Computing can help companies to achieve more efficient use of their IT hardware and software investment and accelerate the adoption of innovation. When making decisions on adopting Cloud Computing organization looks reluctant for many reasons. So there is a necessity to examine the reason or concern for adoption of Cloud Computing services.

\section{MOTIVATION}

As per litterer review we found that Cloud Computing provides many advantages like scalability, cost-reduction, integration but still many organizations/industries/consumers are reluctant to adopt Cloud Computing services. To investigate the reason we carried out this internet based survey study as it has the following advantages:

- $\quad$ Easy: It is easy or convenient method to design and conduct the study with the help of Google forms. It can also quantify our recorded data.

- Cost effective: There is no cost occur during this study during the design and circulate the survey/tool.

- Select audience: On the base of convenience sampling (a type of data collection method) we can easily choose the target audience with the help social networking site and email addresses.

\section{METHODOLOGY}

For achieve this objective we have designed a questionnaire for primary data collection based on convenient sampling into three segments such as IT industry, Education Industry and individual consumers from different organizations.

\subsection{Sources Data Collection}

Secondary Data: Data collection from the printed or published source such as books, journal, research papers, articles and newspapers falls under Secondary data. It is the easy way of data collection. Secondary data analysis also saves the time, provides larger and high quality databases that would be not be easy for any individual researcher to collect on his/her own.

Primary Data: Primary data is directly collected from the respondents with the help of questionnaire. For primary data collection, we have used online questionnaire for which there is a web based survey tool called "RELUCTANCE IN ADOPTION OF CLOUD COMPUTING“. We prepared the questionnaire keeping in mind our objectives. It is cost effective, less time consuming, easy to use and easy to analyze the results. There is no limit for number of questions. To analyze our results as per our requirement, firstly we 
quantify the data and generate Pie charts and Bar Charts.

\subsection{Designing Questionnaire}

Designing questionnaire is a most important task. We prepared questionnaire on the basis of conclusion in the literature review in order to achieve our objectives. We prepared the questionnaire in such a way as to wrap up our research questions in a precise manner. After designing a questionnaire, we have made a pilot study of 30 respondents to check the results. After compilation of whole result of pilot study we finalized our questionnaire for a full fledged survey.

\subsection{Targeted Population}

After literature review, on the basis of our objectives we have arrived at the decision to target small and middle scale IT industries, Education industries, some consumers from different non-IT industries and also those who are directly and indirectly connected with internet under the categories of 'others'. Within these industries we specially targeted IT personnel, network administrators, software developers, faculty, directors, analysts, sever administrators and individual consumers etc. for this survey. While cloud computing is in nascent stage and it is not so common among the various companies and consumers, so to find out the right people who are conversant with cloud computing was a difficult task.

\subsection{Research Objectives}

This survey questionnaire has been designed with following objectives:

- To evaluate the industry specific response on awareness about Cloud Computing

- To explore the industry specific response for willingness to use/adopt cloud computing for (parts of) IT.

- To analyse factors responsible for reluctance to adopt Cloud Computing in general.

\section{SURVEY RESULTS}

As this survey is mainly fucused on evaluate the factors that affect the adoption of cloud computing in the organisations. Our survey questionnaire was designed to investigate how various security threats such as threats in the network, security threat on data, security threat in the web and any other type of security threats impact the adoption of cloud computing. Also, to evaluatethe level of awareness about the cloud computing among the consumers. The Survey result using graphs and charts is as follows:

Q1. What does Cloud Computing primarily mean for your organization (As per the IT, Education and Other industries).

As conclustion of all data collection by the different industries (IT and Education) and individual consumers from different organisations.

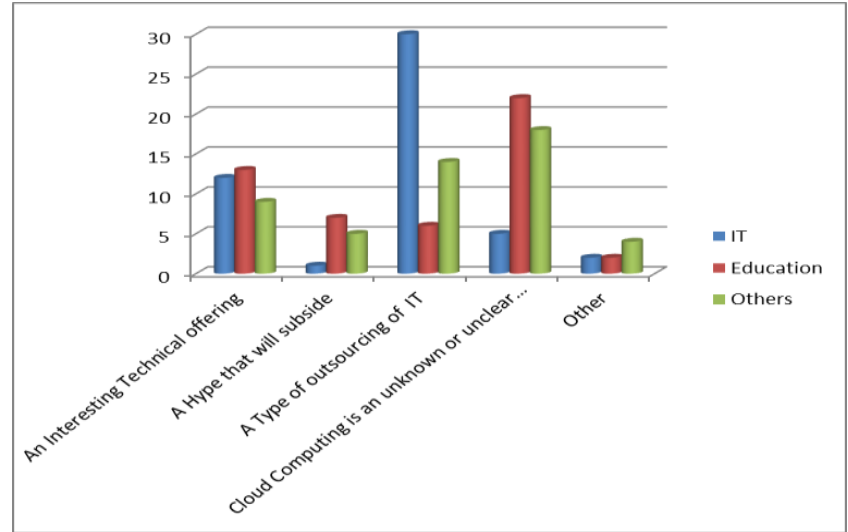

Figure 1. Cloud Computing Understanding

Q2. Is your organisation willing to use/adopt cloud computing for (parts of) your IT (For IT, Education and Other industries)?

As a conclusion of all views:

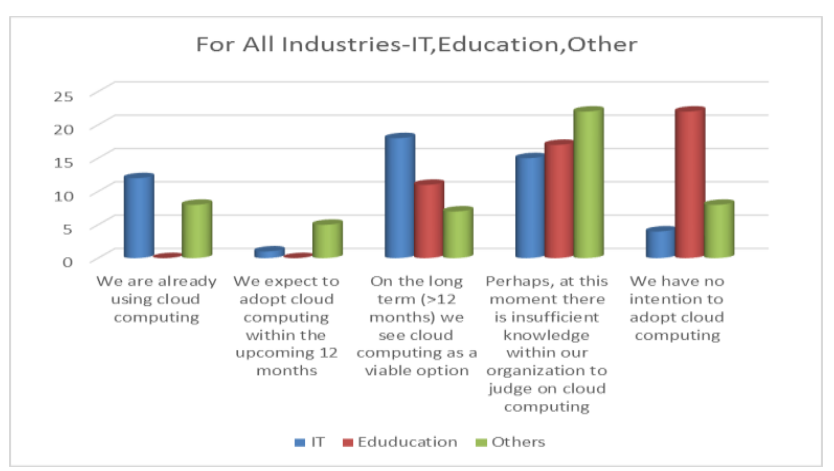

Figure 2. Willing to use/adopt cloud computing

Q3. What are your main concerns regarding the use of cloud computing?

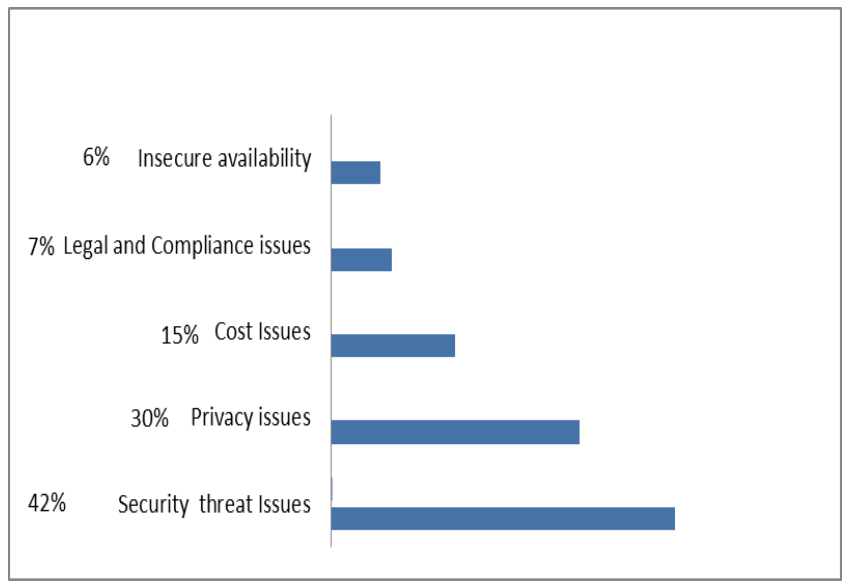

Figure 3. Concern regarding use of cloud computing

Q4. What are your main Concerns related to the Security \& Privacy in the Cloud Computing? 


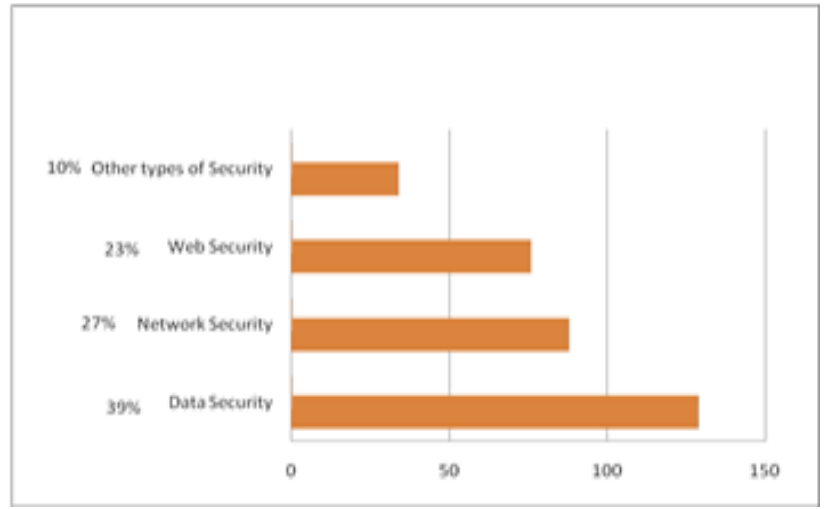

Figure 4. Concern regarding Security \& Threats on cloud computing

Q5. What aspects of cloud computing should be improved?

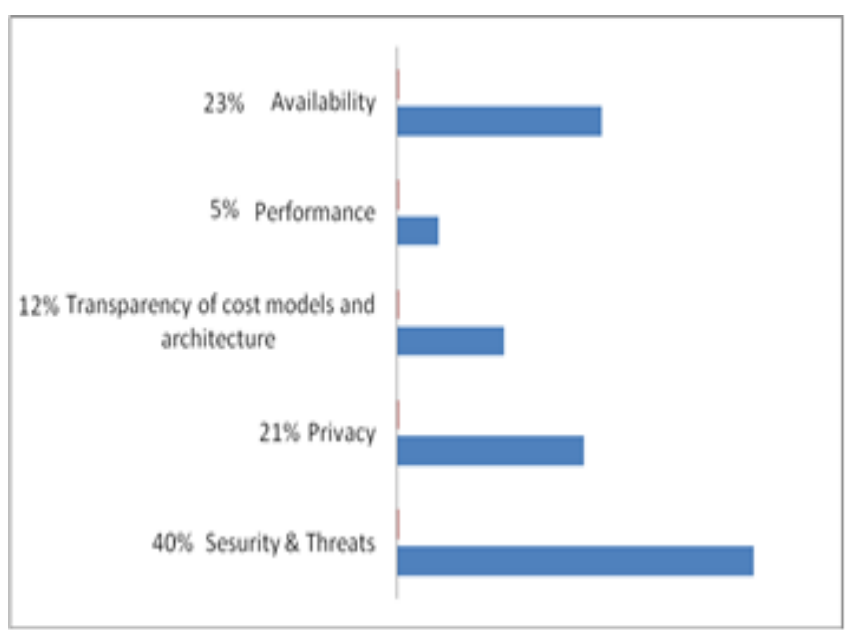

Figure 5. Improved aspects of cloud computing

Q6. What benefits do you expect from cloud computing?

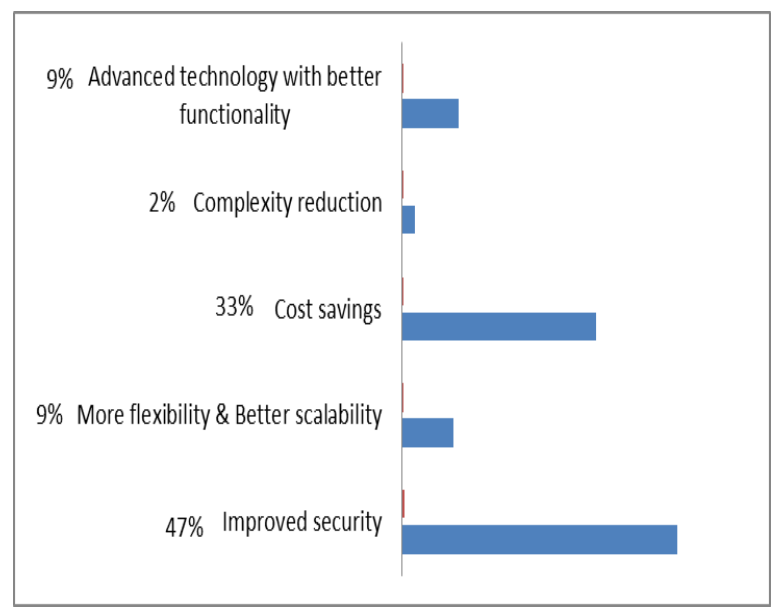

Figure 6. Expected benefits from cloud computing

\section{RESULT INTERPRETATION}

The interpretation of the survey results indicates that while the awareness level about cloud computing is satisfactory among most of the IT indusrty consumers, there is a lack of awareness about cloud computing technology among the employees of Education industry and other Non-IT industries. There is no clear understanding about the capabilities and tenets of Cloud Computing among the employees of Education industry and other Non-IT industries which is not a healthy sign for future proliferation of this powerful technology in general and the business proposions for cloud service providers in particular.

Our next question was to know the opinion about adoption of cloud computing that brought forth an encouraging fact that a high percentage of IT industries believe Cloud Computing as a viable options for adoption on a long term (>12 months) perspective. It also brought out that 'perhaps, at this moment there is insufficient knowledge within our organization to judge on cloud computing'while some of the IT industries are using cloud computing to their advantage. It was revealed in contrast to IT industry that non of the institutions surveyed in education industries was using cloud service, nor they had any intentions of adopting cloud computing. Such a situation could be attributed to unsatisfactory level of awareness for about cloud computing and also ability to take informed decision.

Through the responses to item no $3 \& 4$, an inference could be drawn about the reseans for not adopting cloud computing. It was revealed that the single most important factor resisting the adoption is concern for security issues with $42 \%$ strongly agreeing and more than $70 \%$ agreeing to this statement.

Item number 5 was posed to furhter investigate on what item 3 and 4 had revealed. In other words, under securty concerns whether data security was most prominent concern or the network security. Nearly $40 \%$ consumers showed Data security as their most prominent concern whereas network security was percieved as most prominent concern by 27 percent of the respondents. There were some other concerns alsoas depicted in figure 22 that contributed to adoption of cloud how ever these remain less significant.

Item number 6 and 7 were designed to find out the areas that require attention in order to enhence the adoption of cloud computing at organisational level in future. The interpretation of the results reveal that there is an urgent need to improve and strenghten the Security of the data and privacy of data with visible cost savings. It is understood that only when the consumer is confident of the security and privacy of his/her data and security of the could network, he/she would be ready to migrate to cloud to reap cost benefits of the technology. It is also envisaged that cloud service providers must follow the best practices as a confidence building measure for the client who is worried about the security and privacy of data.

\section{CONCLUSION}

Our result interpretation is revealed that there is large gap in understanding the cloud computing technology among consumers. Specially the majority of the education industries and non-IT industries are unclear and unknown about the cloud computing technology.Majority of consumers perceive that cloud computing is not secure and security is the main blocking factor to limit the adoption of cloud services. The network security threat is of immense concern followed by data security concerns. Lower cost is only positive consideration for implementation of cloud services in organizations.

\section{FUTURE WORK\& SUGGESTIONS}

As an online survey has been conducted to investigate into the reasons of reluctance by different industries such as IT industry, Education industry and non-IT industries. The survey questionnaire consisted questions on cloud awareness 
and cloud security related issues. Some useful insights that have emerged as the interpretation of the survey results, could be put to good use by both the cloud service providers and industries to enhance adoption of cloud computing in future. As well as there is a need to conduct awareness programs, inhouse training programs, improve confidentiality, integrity and availability, improve network security through collaboration, improvement of web-security, improvement of authentication \& authorization process to increase the adoption of cloud computing.

\section{REFERENCES}

[1] Osama Harfoushi, Bader Alfawwaz, Nazeeh A. Ghatasheh, Ruba Obiedat, Mua'ad M. Abu Faraj, and Hossam Faris, "Data Security Issues and Challenges in Cloud Computing: A Conceptual Analysis and Review" Scientific Research, Communications and Network, Published Online February 2014. http://www.scirp.org/journal/cn, http://dx.doi.org/10.4236/cn.2014.61003, 2014, 6, 15-21.

[2] F. Gens, "New IDC It Cloud Services Survey: Top Benefitsand 2009.http://blogs.idc.com/ie/?p=730

Challenges,"

[3] Varsha, Amit Wadhwa and Swati Gupta, "Study of Security Issues in Cloud Computing", International Journal of Computer Science and Mobile Computing (IJCSMC), ISSN: 2320-088X, Volume 4, Issue 6, Page 230-234, June-2015, www.ijcsmc.com.

[4] B. Sumitra, C. R. Pethuru, and M. Misbahuddin, "A Survey of Cloud Authentication Attacks and Solution Approaches", International Journal of Innovative Research in Computer and Communication Engineering (IJIRCCE), An ISO 3297:2007, ISSN (online): 2320-
9801, ISSN (Print): 2320-9798, Volume 2, Issue 10, October 2014, www.ijircce.com.

[5] Chiraj Modi, Dhiren Patel, Bhavesh borisaniya, Avi Patel, and Muttukrishnan Rajarajan, "A Survey On Security Issues and Solutions At different layers of Cloud Computing", The Journal of Supercomputing, Volume 63 Issue 2, February 2013.

[6] Rajesh Piplode, and Umesh Kumar Singh, "An overview and Study of Security Issues \& Challenges in Cloud Computing", International Journal of Advanced Research in Computer Science and Software Engineering, ISSN: 2277 128X, Volume 2, Issue 9, September 2012.

[7] AjithSingh. N, Vasanthi. V and M. Hemalatha, "A Brief Survey on Architecture, Challenges \& Security Benefit in Cloud Computing", International Journal of Information and Communication Technology Research, Volume 2 No 2, Page No-102 and 109, February 2012.

[8] Wayne A. Jansen, "Cloud Hooks: Security and Privacy Issues in Cloud Computing", Proceeding of the $4^{\text {th }}$ Hawaii International Conference on System Sciences, 2011.

[9] S. Subashini and V. Kavitha, "A Survey on Security Issues in service delivery Models of Cloud Computing", Jouranal of Network and Computer applications 34 (2011), Elsevier, January 2011.

[10] Jeff Bardin, Jon Callas, Privity Pam Fusco, Francoise Gilbert, Christofer Hoff, Dennis Hurst, Subra Kumaraswamy, and Liam Lynch, "Security Guidance for Critical Areas of Focus in Cloud Computing", https://cloudsecurityalliance.org/guidance/csaguide.v1.0. pdf, April 2009. 